\title{
Study of the Dispersive Component of the Surface Energy of Polylactides by Inverse Gas Chromatography at Infinite Dilution
}

\author{
Carla Zarazir1,2, Mohamad Rajab1,2, Houssam Obeid1,2, Joumana Toufaily ${ }^{1,2}$, Imad Toufeili³, \\ Tayssir Hamieh1,2*
}

${ }^{1}$ Laboratory of Materials, Catalysis, Environment and Analytical Methods Laboratory (MCEMA), Lebanese University, Hadath, Lebanon

${ }^{2}$ Laboratory of Applied Studies to the Sustainable Development and Renewable Energies (LEADDER), EDST, Lebanese

University, Hadath, Lebanon

${ }^{3}$ Department of Nutrition and Food Sciences, American University of Beirut (AUB), Beirut, Lebanon

Email: ^tayssir.hamieh@ul.edu.lb

How to cite this paper: Zarazir, C., Rajab, M., Obeid, H., Toufaily, J., Toufeili, I. and Hamieh, T. (2020) Study of the Dispersive Component of the Surface Energy of Polylactides by Inverse Gas Chromatography at Infinite Dilution. American Journal of Analytical Chemistry, 11, 129-136.

https://doi.org/10.4236/ajac.2020.113010

Received: January 27, 2020

Accepted: February 28, 2020

Published: March 3, 2020

Copyright $(2020$ by author(s) and Scientific Research Publishing Inc. This work is licensed under the Creative Commons Attribution International License (CC BY 4.0).

http://creativecommons.org/licenses/by/4.0/

(c) (i) Open Access

\begin{abstract}
Inverse gas chromatography at infinite dilution is a powerful technique that can be advantageously used to characterize the surface physicochemical properties of solid substrates as oxides and polymers in both forms of powder or fibres. In the case of polymer, this technique can be used to determine the second order transition phenomena temperatures. This paper was devoted to the determination of the glass transition temperature of polylactide polymer. The dispersive component of the surface energy $\gamma_{s}^{d}$ of polylactides was determined by inverse gas chromatography at infinite dilution. Various theoretical models were used to deduce the dispersive component of the surface energy of the solid substrates. These models are based on the calculation of the molecular areas of adsorbed molecules on the polymer surface: geometrical model, cylindrical molecular model, liquid density model, BET method, Kiselev results and the two-dimensional Van der Waals and Redlich-Kwong equations. The curves relative to the variation of $\gamma_{S}^{d}$ as a function of the temperature showed a specific graph with a maximum value of $\gamma_{s}^{d}$ at a certain particular temperature characteristic to the investigated polylactide polymer. In fact, the maxima of $\gamma_{S}^{d}$ indicated the presence of glass transition temperature $\mathrm{Tg}$ of polylactide whatever the molecular model used. This study showed a glass transition temperature equal to $64^{\circ} \mathrm{C}$ confirming that obtained by other studies.
\end{abstract}

\section{Keywords}

Adsorption, Surface Area, Surface Energy, Retention Volume, N-Alkanes, 
Equations of State

\section{Introduction}

The inverse gas chromatography (IGC) at infinite dilution was proved to be an important technique to determine the surface properties of solid materials [1] [2] [3] [4]. This technique was used for more than thirty years to determine the physico-chemical surface properties of solid materials or nanomaterials, polymers, oxides or polymers adsorbed on oxides. One of the interesting properties is the dispersive component of the surface energy of solid materials that can be determined as a function of the temperature.

In the IGC technique at infinite dilution, model organic molecules of known properties are injected in the column containing the solid. The retention times tn of these molecules, measured at infinite dilution, allow us to determine retention volume $V n$ and therefore the interactions between the model polar or non-polar molecules and the solid substrates, by supposing that there is no lateral interaction between the probe molecules themselves. Measurements can be carried out with a GC FID chromatograph equipped with a flame ionization detector of high sensitivity. The data retention can be obtained with a stainless steel column with specific dimensions packed with $1-2 \mathrm{~g}$ of solids in powder forms.

The dispersive component of the surface energy of solids $\gamma_{s}^{d}$ can be in general calculated using the equation of the dispersive free energy of adsorption $\Delta G^{d}$ given as a function of the geometric mean of the dispersive components (exponent d) of the surface energy of the probe $\gamma_{1}^{d}$ and the solid $\gamma_{s}^{d}$ :

$$
\Delta G^{d}=R \ln V n+C=\mathcal{N} a W_{a}=2 \mathcal{N} a\left(\gamma_{l}^{d} \gamma_{s}^{d}\right)^{1 / 2}
$$

where $R$ is the ideal gas constant, $T$ the absolute temperature and $C$ a constant depending on the reference state of adsorption, $W_{a}$ is the energy of adhesion, $\mathcal{N}$ is Avogadro's number and a the surface area of one adsorbed molecule of the probe.

By plotting $R T \ln V n$ as a function of $\mathcal{N a}\left(\gamma_{l}^{d}\right)^{1 / 2}$ of $\mathrm{n}$-alkanes, it is possible to deduce, from the slope of the straight line, the value of dispersive component $\gamma_{S}^{d}$ of the surface energy of the solid material.

However, the values of the surface area of organic molecules adsorbed on a solid substrate are not known with a good accuracy, especially because of the change of molecule positions when approaching a solid surface at a certain temperature. The problem will be more complicated when the temperature increases; in such case, we cannot neglect the effect of the thermal agitation on the surface areas of adsorbed molecules. In a previous study, Hamieh et al. [5] proved the effect of the temperature on the surface area of n-alkanes and polar molecules. In this study, we proposed various theoretical models giving the molecular areas of n-alkanes: geometrical model, cylindrical molecular model, liquid density model, BET method, Kiselev results and the two-dimensional Van der Waals 
model by using its constant $b$ that depends on the critical temperature and pressure of the liquid. The Redlich-Kwong equation transposed from three-dimensional space to two-dimensional space [6] [7] [8] was also used to calculate the areas of organic molecules. Table 1 summarizes the different surface area values for the used $n$-alkanes using the various molecular models.

Hamieh et al. [5] showed the areas a(T) of molecules adsorbed on Polytetrafluoroethylene (PTFE), linearly depend of the temperature. The following relation was proved:

$$
a(T)=a_{0}-\Omega T
$$

with $\Omega$ the slope of the straight line depending on the nature of the adsorbed molecule and solid substrate, $a(T)$ the surface area at temperature $T$ and $a_{0}$ the molecule area extrapolated at $0 \mathrm{~K}$.

Therefore, to have the behavior of the dispersive component of the surface energy of any solid material, we need to apply the above theoretical models, given the different surface areas of the adsorbed molecules. Consequently, it will be possible to determine different values of $\gamma_{S}^{d}$ of the same solid at a fixed temperature depending on the model used.

Knowing that there is an important effect of the temperature on the polymer properties. This can induce modifications in the chain segment mobility of polymers. These changes in mobility arising at the glass transition temperatures (Tg) of bulk polymers can be determined by using the thermal methods, like differential thermal analysis or differential scanning calorimetry.

However, for smaller quantities of polymer, the glass transitions of polymers could not be detected by these previous thermal methods. To remediate this problem, we used the inverse gas chromatography at infinite dilution to obtain more precise information on the transition phenomenon temperatures.

In this paper, we propose to use the inverse gas chromatography at infinite dilution to determine the glass transition temperature of polylactide polymer. This technique will allow to determine the dispersive component of the surface energy of polylactide polymer (PLA) that has been playing a major role in many applications such as medical science, biotechnology and food science due to their biocompatibility and biodegradability [9]. These polymers can be obtained

Table 1. Surface areas of various molecules (in $\AA 2$ ) obtained from the various models of Van der Waals (VDW), Redlich-Kwong (R-K), Kiselev, geometric, cylindrical or spherical models.

\begin{tabular}{ccccccc}
\hline Molecule & VDW & Kiselev & Cylindrical & R-K & Spherical & Geometric \\
\hline $\mathrm{C}_{5} \mathrm{H}_{12}$ & 47.0 & 45 & 39.3 & 36.8 & 36.4 & 32.9 \\
$\mathrm{C}_{6} \mathrm{H}_{14}$ & 52.7 & 51.5 & 45.5 & 41.3 & 39.6 & 40.7 \\
$\mathrm{C}_{7} \mathrm{H}_{16}$ & 59.2 & 57 & 51.8 & 46.4 & 42.7 & 48.5 \\
$\mathrm{C}_{8} \mathrm{H}_{18}$ & 64.9 & 63 & 58.1 & 50.8 & 45.7 & 56.2 \\
$\mathrm{C}_{9} \mathrm{H}_{20}$ & 69.6 & 69 & 64.4 & 54.5 & 48.7 & 64.0 \\
\hline
\end{tabular}


from a renewable agricultural source such as corn or starch, they provide significant energy savings, they are recyclable and compostable and they have the ability to decompose into naturally occurring metabolites via hydrolysis or enzymatic processes [10] [11].

The determination of the dispersive component of the surface energy of polylactide polymer against the temperature will allow to evaluate the glass transition temperature of the polymer. In the next section we develop the experimental part necessary to quantify the surface properties of PLA polymer.

\section{Experimental Part}

\subsection{Retention Volume}

The net retention volume $V n$ represents the volume of carrier gas through the column since the introduction of the probe until the output of the maximum of the peak of the inferred methane retention volume $V_{0}$. It is influenced by the retention time according to the following relation:

$$
V n=j D_{c}\left(t_{R}-t_{0}\right)
$$

where $t_{R}$ is the retention time of the probe, $t_{0}$ the zero retention reference time measured with a non-adsorbing probe such as methane, $D_{c}$ the flow rate and $j$ a correction factor taking into account the compression of the gas [12]. $D_{c}$ and $j$ are respectively given by the following expressions:

$$
D_{c}=D_{m} \frac{T_{c}}{T_{a}} \frac{\eta\left(T_{c}\right)}{\eta\left(T_{a}\right)}
$$

and

$$
j=\frac{3}{2} \frac{\left(\frac{\Delta P+P_{0}}{P_{0}}\right)^{2}-1}{\left(\frac{\Delta P+P_{0}}{P_{0}}\right)^{3}-1}
$$

where $D_{m}$ is the measured flow rate, $T_{c}$ the column temperature, $T_{a}$ the room temperature, $\eta(T)$ the gas viscosity at temperature $T, P_{0}$ the atmospheric pressure and $\Delta P$ the pressure variation.

\subsection{Materials}

PLA polymers used in the storage of olive oils were procured directly from Lebanese markets. The packaging polymer samples were cut into small rectangular pieces with scissors and using cotton gloves to avoid contamination of the plastic samples. The area of each test specimen was equal to $10 \mathrm{~cm}^{2}$. The polymer pieces were then grinded to obtain a size range of PLA particles between 100 and 250 $\mu \mathrm{m}$. Particles of the correct size were introduced into a stainless steel column, which was $30 \mathrm{~cm}$ long and had an internal diameter of $2 \mathrm{~mm}$. A mass of $1 \mathrm{~g}$ of PLA was used to fill the chromatographic column. The column filled with the sample was conditioned at $120^{\circ} \mathrm{C}$ for $12 \mathrm{~h}$ to remove any impurities. 
The measurements of retention time were done by using the IGC technique. The column was then attached to the gas chromatograph, fitted with a flame ionization detector. Helium was used as carrier gas; its flow-rate was equal to about $20 \mathrm{~mL} \cdot \mathrm{min}^{-1}$. IGC measurements at infinite dilution were done by varying the temperature from $40^{\circ} \mathrm{C}$ to $100^{\circ} \mathrm{C}$.

Chromatographic injections were made using Hamilton microsyringes. We used the inverse gas chromatography technique at infinite dilution for the physicochemical characterization of different materials. Measurements were carried out with a DELSI GC121 FB Chromatograph equipped with a flame ionization detector of high sensitivity.

Classical organic probes as n-alkanes with highly pure grade (Aldrich, i.e., 99\%) were used for IGC measurements. The probes used were n-alkanes such as pentane, hexane, heptane, octane, and nonane.

\subsection{GC Conditions}

The IGC measurements were performed on a commercial Shimadzu GC-14A gas chromatograph equipped with a flame ionization detector. Dried nitrogen was the carrier gas. The gas flow rate was set at $20 \mathrm{~mL} / \mathrm{min}$. The injector and detector temperatures were maintained at $200^{\circ} \mathrm{C}$ during the experiments. To achieve infinite dilution, $0.5 \mu \mathrm{L}$ of each probe was injected with $10 \mu \mathrm{L}$ Hamilton syringes, in order to approach linear condition gas chromatography. The column temperatures were $40^{\circ} \mathrm{C}$ to $100^{\circ} \mathrm{C}$, varied in $5^{\circ} \mathrm{C}$ to $10^{\circ} \mathrm{C}$ steps. Each probe injection was repeated three times, and the average retention time, $t_{R}$, was used for the calculation. The standard deviation was less than $1 \%$ in all measurements.

The columns used in this study were prepared using a stainless-steel column with a $5 \mathrm{~mm}$ inner diameter and with an approximate length of $30 \mathrm{~cm}$.

\subsection{Results and Discussion}

\section{Determination of the dispersive component of the surface energy of PLA}

We applied the different models of the surface area of $\mathrm{n}$-alkanes given in Table 1 , in order to obtain qualitative results relative to the dispersive component of the surface energy of PLA polymer used in this study. Table 1 and Equation (1) allowed us to determine the variations of $\gamma_{S}^{d}$ of PLA polymer as a function of the temperature that can determine the glass transition temperature of PLA surface.

Results obtained by this study can be gathered in Table 2 .

Using the results of Table 2, we plotted on Figure 1 the variation of the dispersive component of the surface energy of PLA polymer, as a function of the temperature. Figure 1 shows a change in the variation of $\gamma_{s}^{d}$ versus the temperature. It seems that the maxima of the curves correspond to glass transition temperature $\left(\mathrm{Tg}=337 \mathrm{~K}\right.$ or $\left.64^{\circ} \mathrm{C}\right)$ of the polymer for all models of the surface area of n-alkanes. 
Table 2. Values of $\gamma_{S}^{d}\left(\mathrm{~mJ} / \mathrm{m}^{2}\right)$ of PLA surface using different models of molecular model of surface area of n-alkanes.

\begin{tabular}{ccccccc}
\hline T (K) & VDW & Kiselev & Cylindrical & R-K & Spherical & geometric \\
\hline 313 & 23.8 & 25.0 & 24.2 & 39.5 & 59.6 & 18.8 \\
323 & 20.0 & 20.8 & 20.4 & 33.2 & 52.6 & 14.8 \\
333 & 18.7 & 19.7 & 19.3 & 29.4 & 48.4 & 13.4 \\
335 & 21.8 & 22.6 & 22.2 & 37.4 & 56.9 & 16.8 \\
337 & 25.0 & 26.6 & 25.7 & 43.1 & 66.5 & 20.1 \\
340 & 21.6 & 23.4 & 22.8 & 36.1 & 55.2 & 16.1 \\
343 & 19.3 & 20.1 & 19.7 & 28.6 & 46.3 & 13.2 \\
353 & 17.6 & 18.7 & 18.6 & 25.1 & 40.5 & 12.9 \\
363 & 15.6 & 16.9 & 16.5 & 22.5 & 34.2 & 12.0 \\
373 & 14.8 & 15.8 & 15.5 & 20.4 & 30.2 & 11.7 \\
\hline
\end{tabular}

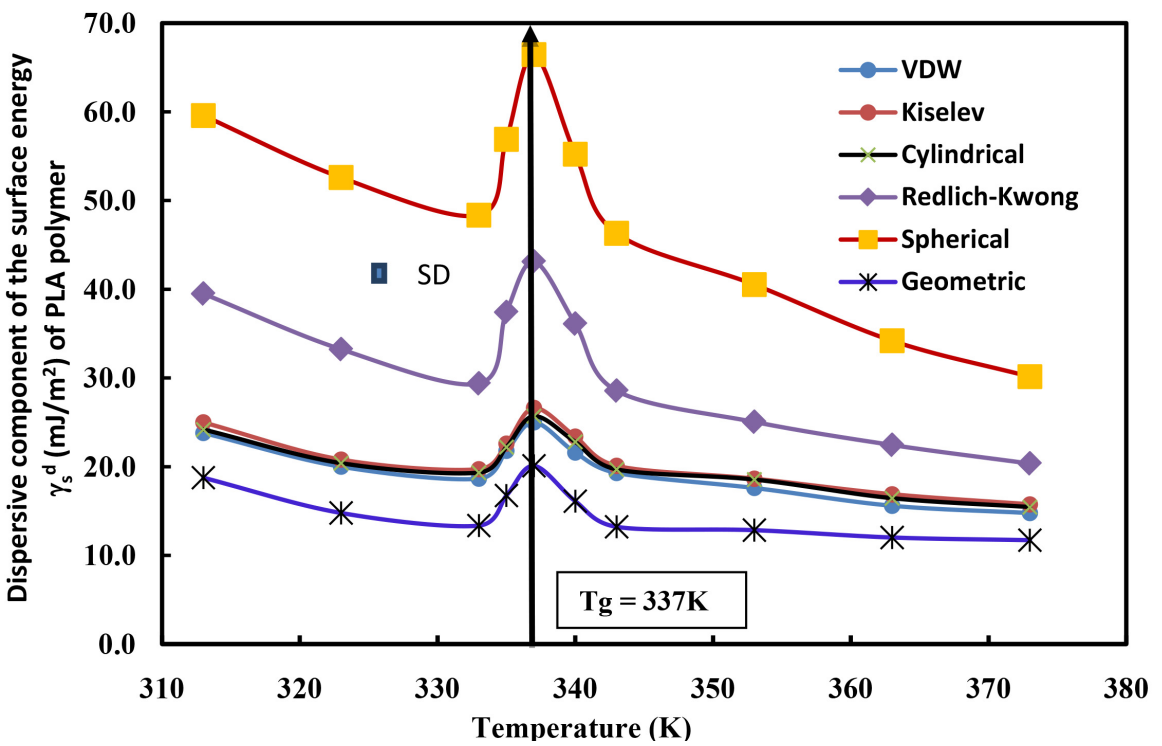

Figure 1. Evolution of the dispersive component of the surface energy of polylactide polymer as a function of the temperature for different models of surface areas of n-alkanes.

This result $\left(\mathrm{Tg}=64^{\circ} \mathrm{C}\right)$ confirmed that obtained in a previous study [13] on PLA we using the retention volume of different $n$-alkanes adsorbed on PLA polymers. The curves plotted on Figure 2 of $R T \ln V n=f(1 / T)$ of PLA for the different $\mathrm{n}$-alkanes showed the presence of a glass transition $\mathrm{Tg}=64^{\circ} \mathrm{C}$ (on Figure $\left.2,1 / \mathrm{Tg}=0.00297 \mathrm{~K}^{-1}\right)$.

\section{Discussion and Conclusion}

In this paper, we determined the dispersive component of the surface energy of polylactide polymer by using the inverse gas chromatography technique at infinite dilution for different models of surface areas of $n$-alkanes. It is the first time 


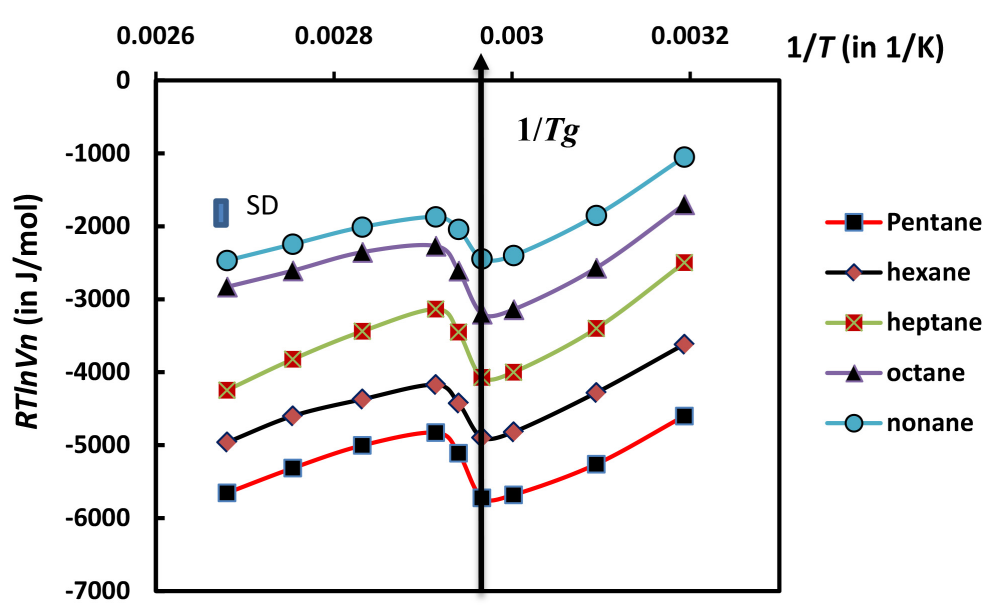

Figure 2. Variation of specific $R T \ln V n$ (in $\mathrm{J} / \mathrm{mol}$ ) of n-alkanes adsorbed on PLA polymer as a function of the inverse of the temperature $(1 / \mathrm{T})$.

that we obtained the glass transition temperature $\left(\mathrm{Tg}=64^{\circ} \mathrm{C}\right)$ of PLA by using the evolution of $\gamma_{S}^{d}$ as a function of the temperature. This method confirmed the value of Tg of PLA by another study.

The maxima of $\gamma_{s}^{d}$ indicate in Figure 1 the presence of glass transition temperature. The change in retention mechanism, of the probes, at the transition temperature is attributed to an increased molecular mobility of the polymer segments, allowing for the penetration of the probes into the polymer layer. Indeed, for polymer below $\mathrm{Tg}$, the penetration of the solute molecules in the bulk polymer is precluded and retention proceeds only by surface adsorption. Then, at glass transition, the penetration of the probe molecules begins but due to an initially slow rate of diffusion of the solute into and out of the polymer, non-equilibrium conditions prevail. This can explain the decrease in the retention volume with temperature at $\mathrm{Tg}$. As temperature is increased, the diffusion coefficient rises sharply which leads to equilibrium conditions. It can be observed that the maximum of $\gamma_{S}^{d}$ corresponds to the minimum of the curves of the evolution of $R T I n V n$ as a function of $(1 / \mathrm{T})$ as was shown in Figure 2.

\section{Funding Sources}

This research did not receive any specific grant from funding agencies in the public, commercial, or not-for-profit sectors.

\section{Conflicts of Interest}

The authors declare no conflicts of interest regarding the publication of this paper.

\section{References}

[1] Ticehurst, M.D. (1995) Characterisation of the Surface Energetics of Pharmaceutical Powders by Inverse Gas Chromatography. PhD Thesis, University of Bradford, York. 
[2] Buckton, G. (1997) Characterisation of Small Changes in the Physical Properties of Powders of Significance for Dry Powder Inhaler Formulations. Advanced Drug Delivery Reviews, 26, 17-27. https://doi.org/10.1016/S0169-409X(97)00507-3

[3] Al-Ghamdi, A. and Al-Saigh, Z.Y. (2002) Surface and Thermodynamic Characterization of Conducting Polymers by Inverse Gas Chromatography. I. Polyaniline. Journal of Chromatography $A, 969,229-243$. https://doi.org/10.1016/S0021-9673(02)00887-7

[4] Que, R., Wu, D. and Al-Saigh, Z.Y. (2007) Surface and Thermodynamic Characterization of Conducting Polymers by Inverse Gas Chromatography: II. Polyaniline and Its Blend. Journal of Chromatography A, 1146, 93-102.

https://doi.org/10.1016/j.chroma.2007.01.093

[5] Hamieh, T. and Schultz, J. (1996) Etude par chromatographie gazeuse inverse de l'influence de la température sur l'aire de molécules adsorbées. The Journal of Chemical Physics, 93, 1292-1331. https://doi.org/10.1051/jcp/1996931292

[6] Hamieh, T., Rezzaki, M. and Schultz, J. (2001) Study of the Second Order Transitions and Acid-Base Properties of Polymers Adsorbed on Oxides, by Using Inverse Gas Chromatography at Infinite Dilution, II Experimental Results. Journal of Colloid and Interface Science, 233, 343-347. https://doi.org/10.1006/jcis.2000.7271

[7] Hamieh, T., Rezzaki, M. and Schultz, J. (2001) Study of the Transition Temperatures and Acid-Base Properties of Poly(methyl methacrylate) Adsorbed on Alumina and Silica, by Using Inverse Gas Chromatography Technique. Colloids and Surfaces A: Physicochemical and Engineering Aspects, 189, 279-291. https://doi.org/10.1016/S0927-7757(01)00597-0

[8] Hamieh, T. (2011) Determination of the Transition Phenomena of Poly ( $\alpha$-n-alkyl) Methacrylates Adsorbed on Silica by Inverse Gas Chromatography (IGC). Journal of Polymer Research, 18, 1159-1168. https://doi.org/10.1007/s10965-010-9519-9

[9] Ahmed, J. and Varshney, S.K. (2011) Polylactides-Chemistry, Properties and Green Packaging Technology: A Review. International Journal of Food Properties, 14, 37-58. https://doi.org/10.1080/10942910903125284

[10] Saltzman, W.M. (2001) Drug Delivery: Engineering Principles for Drug Therapy. Oxford University Press, New York, 334-336.

[11] Auras, R., Harte, B. and Selke, S. (2004) An Overview of Polylactides as Packaging Materials. Macromolecular Bioscience, 4, 835-864. https://doi.org/10.1002/mabi.200400043

[12] Gutmann, V. (1978) The Donor-Acceptor Approach to Molecular Interactions. Plenum, New York. https://doi.org/10.1007/978-1-4615-8825-2

[13] Zarazir, C., Rajab, M., Obeid, H., Toufaily, J., Toufeili, I. and Hamieh, T. (2020) New Methods to Characterize the Surface and Interface Acid-Base Properties of Polylactides Polymers by Inverse Gas Chromatography. Chromatography and Separation Techniques Journal. 